Pulsar Astronomy - 2000 and Beyond

ASP Conference Series, Vol. 202, 2000

M. Kramer, N. Wex, and R. Wielebinski, eds.

\title{
About the Magnetic Field Generation in Crab Nebula
}

\author{
Irakli S. Nanobashvili \\ Abastumani Astrophysical Observatory, Georgian Academy of Sciences, \\ A. Kazbegi Ave.2a, GE-380060 Tbilisi, Georgia
}

\begin{abstract}
The new plasma mechanism for the magnetic field generation in Crab Nebula is suggested. The mechanism is based on the development of two-stream instability after the supernova explosion.
\end{abstract}

\section{Introduction}

Crab Nebula is one of the most interesting object in the universe. It is the remnant of the supernova which exploded in 1054. From this nebula has been detected for the first time the nonthermal type radiation. Shklovsky suggested that this radiation can be explained by the synchrotron mechanism (Shklovsky 1953), which was proved successfully by the observations of Vashakidze in Abastumani (Vashakidze 1954). For the generation of this radiation it is necessary the existence of relativistic charged particles and strong magnetic field in Crab Nebula. The problem of the relativistic charged particles has been solved after the discovery of pulsar inside the Crab Nebula - these particles are brought in the nebula by the relativistic pulsar wind. As for the generation of magnetic field, this probem is not solved finally yet. Several mechanisms have been suggested (see example Piddington 1957, Kennel \& Coroniti 1984, Kennel \& Coroniti 1984, Tsikarishvili et al. 1988), but practically all these mechanisms require the existence of the outer shell in Crab Nebula, which is unpenetrable for the relativistic charged particles and magnetic field. Observations have not proved the existence of this shell.

We propose the new plasma mechanism for the generation of the magnetic field in Crab Nebula, which is based on the development of two-stream instability after the supernova expolsion.

\section{Main consideration}

As we have mentioned above Crab Nebula is the remnant of the supernova which exloded in 1054. During the explosion plasma of the outer layers of presupernova has been thrown radially outwards against the interstellar plasma. Let us discuss the dynamics of electromagnetic perturbations in such plasma, which consists of two plasma streams - plasma of the outer layers of pre-supernova and interstellar plasma moving through each other. For this purpose one needs the equation of the motion, continuity equation and Maxwell equations. For the first time we assume that the unperturbed static magnetic field is zero $-B_{0}=0$. After the standard linearisation procedure finally one can get the dispersion 
relation in the folowing form (see in detail Machabeli et al. 1999):

$$
\omega^{2}=-\omega_{p}^{2} \frac{V_{0}^{2}}{1+\omega_{p}^{2} / k^{2}} .
$$

Here we use geometric units - $c=G=1$. The time dependence of the perturbed magnetic field is the following:

$$
B_{1} \sim \exp (-i \omega t) .
$$

¿From (1) and (2) it is evident that the two-stream instability development, i.e. the generation of the exponentially growing magnetic field takes place in Crab Nebula.

If we discuss the cases when the unperturbed static magnetic field $B_{0}$ is nonzero and it is directed for the first time along and then transverse to the plasma flow, it can be easily found (see in detail Machabeli et al. 1999) that if the following condition is fulfilled

$$
\frac{B_{0}^{2}}{4 \pi n_{0} m V_{0}^{2}}<1
$$

(this condition is really fulfilled in Crab Nebula) the two-stream instability development, i.e. the generation of the exponentially growing magnetic field takes place in Crab Nebula.

\section{Conclusion}

The new plasma mechanism of the magnetic field generation in Crab Nebula is proposed, which is based on the two-stream instability development after the supernova exlosion. The generated magnetic field extracts the energy from the plasma particle kinetic energy, which exceeds by an order of the magnitude the total energy of the magnetic field in Crab Nebula according to the current estimations. So, there is a great source of energy to provide the magnetic field generation in Crab Nebula according to the mechanism suggested by us.

\section{References}

Shklovsky, I.S. 1953, DAN SSSR, 90, 983. (in Russian)

Vashakidze, M.A. 1954, Astron. Circ., No 147. (in Russian)

Piddington, N.S. 1957, Australian J. Phys., 10, 350.

Kennel, C.F. \& Coroniti, F.V. 1984, ApJ, 283, 694.

Kennel, C.F. \& Coroniti, F.V. 1984, ApJ, 710, 694.

Tsikarishvili, E.G., Chagelishvili, G.D., Chedia, O.V., Lominadze, J.G \& Sokhadze, Z.A. 1988, Ap\&SS, 149, 255.

Machabeli, G.Z., Nanobashvili, I.S. \& Tendler, M. 1999, Physica Scripta, accepted for publication. 